
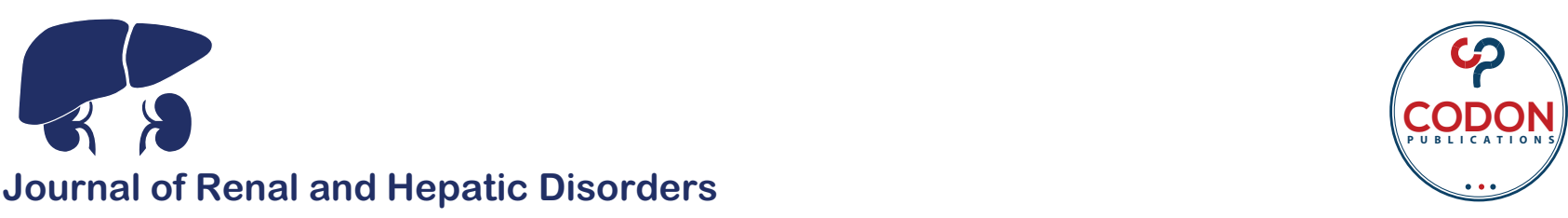

ORIGINAL PAPER/ RESEARCH

\title{
The Effect of Clinical and Demographic Factors on Quality of Life in End-Stage Renal Disease. A Multicenter Cross-Sectional Study
}

\author{
Evangelos C. Fradelos
}

Nursing Department, University of Peloponnese, Sparta, Greece

\begin{abstract}
The aim of this study was to assess the quality of life (QoL) in end-stage renal disease (ESRD) patients undergoing hemodialysis and to examine the clinical and demographic attributes associated with it. This is a multicenter, cross-sectional study, conducted in 2018, in which 367 patients with ESRD undergoing hemodialysis were recruited. Data were collected through a two-part anonymous, self-completed questionnaire. The first part contained questions regarding demographic, social, and clinical information, and the Greek version of Missoula-Vitas Quality of Life Index-15 (MVQoL-15) scale was used to assess QoL. Descriptive and inferential statistical methods were applied. All reported p-values were two-tailed, and the statistical significance level was set at 0.05 . Of the study participants, $62.1 \%$ were male, with a mean age of $61.80 \pm 15.11$. Of the participants, $67.3 \%$ were living in urban areas and $59.1 \%$ were married. The majority of the Hemodialysis (HD) patients evaluated possessively all the domains of MVQoL except the wellbeing Domain. QoL was found to be influenced, among others, by age (0.001), occupation (0.002), education (0.003), and additional health problems $(<0.001)$. The role of patient characteristics in QoL is an area of interest, and early and proper intervention is important to enhance QoL.
\end{abstract}

Keywords: clinical factors; demographic factors; end-stage renal disease; hemodialysis; quality of life

Received: 11 January 2020; Accepted after revision: 16 March 2020; Published: 07 May 2020.

Author for correspondence: Evangelos C. Fradelos, Nursing Department, University of Peloponnese, Sparta, Greece. Email: efradelos@med.uoa.gr

How to cite: Evangelos C. Fradelos. The Effect of Clinical and Demographic Factors on Quality of Life in End-Stage Renal Disease. A Multicenter Cross-Sectional Study. J Ren Hepat Disord. 2020;4(1):1-9.

Doi: http://dx.doi.org/10.15586/jrenhep.2020.58

Copyright: Evangelos C. Fradelos

License: This open access article is licensed under Creative Commons Attribution 4.0 International (CC BY 4.0). http://creativecommons.org/ licenses/by/4.0

\section{Introduction}

The number of patients with chronic kidney disease (CKD) is increasing dramatically. It is estimated that more than 1.4 million people worldwide are undergoing a renal replacement procedure with an annual increase of $8 \%$. Hemodialysis is the most widely used method of kidney replacement, and it was first used in 1945 for the treatment of acute renal failure (1).

Patients on dialysis visit dialysis centers thrice a week for 3-4 h per session, which can affect both their professional and personal lives (2). Every patient with CKD requires a personalized treatment plan to ensure their survival and a satisfactory standard of living. The view of QoL is subjective, with expectations, experiences, behaviors, values and philosophies. Hemodialysis patients account for $88 \%$, and the remaining $12 \%$ undergo other forms of renal replacement therapy. They visit the nearest artificial kidney unit, morning or afternoon. Changes are needed both at the professional level as well as with regard to eating and entertainment habits. As the kidney function decreases, patients needs are increasing and changing (3).

Nowadays, there is a growing interest in the concept of quality of life (QoL). Many researchers, especially in social 
and biomedical sciences, are trying to measure and determine the QoL, reaching the point of seeking safe conclusions through various indicators. These indicators can be used by economists and policymakers to design interventions concerning the economy, environment, social and health policy (4). Evaluating QoL is particularly important, especially in cases where treatment does not deliver the expected results that will contribute to achieving increased patient life expectancy. Therefore, a functional definition of QoL can be a situation in which human life is characterized as a good on the basis of two components: (a) the patient's ability to perform activities that require physical, mental and social well-being and (b) to satisfy the individual with regard to his or her functionality and disease control (5). In addition, QoL can be defined as the functional outcome of the disease and treatment that accompanies it from the perspective of the patients themselves (6).

Assessment of QoL in patients with CKD undergoing hemodialysis is an important aspect of patient care as many patients face chronic health issues (7). The impact of CKD at the physical and psychosocial levels can significantly affect the QoL of the patients and their satisfaction with their daily lives. The disease directly affects the profession and career of those affected, thereby having a negative impact not only on the income required to combat the disease, whether it be peritoneal dialysis or hemodialysis, but also on their social status (8).

Research data support the fact that individual differences can be associated with QoL and the well-being of patients undergoing hemodialysis. Women tend to report reduced QoL as opposed to men. Poorer QoL is often associated with increased age in patients with end-stage renal disease (ESRD) (9). In addition, individuals with a lower socio-economic status report lower QoL and poorer psychological well-being. On the contrary, higher economic and educational levels are associated with higher health-related QoL. Similarly, marital status can be associated with QoL as married individuals seem to be related to better physical health and well-being $(9,10)$.

Additional factors that have been found to affect QoL in ESRD, for example, are educational level and how patients respond to the environment in which they are living. It is also important to change one's habits as therapeutic intervention (e.g., dialysis) will force the individual to come to terms with a number of limitations. Moreover, comorbidities, psychological distress and the various restrictions on daily routine have been associated with poorer QoL (9-11).

Although various researches addressing QoL in hemodialysis patients have been published, there are no multicenter researches addressing the effect of clinical and demographic characteristics in Greece. Thus, the aim of the present study was to evaluate QoL of hemodialysis patients in Greece and to examine the effect of clinical and demographic characteristics on QoL of hemodialysis patients.

\section{Material and Methods}

\section{Study design and sample}

A multicenter, cross-sectional study in five public dialysis units and one private dialysis unit, covering six major regions of Greece, was conducted in 2018. A total of 367 patients with ESRD undergoing hemodialysis thrice a week were recruited. This accounts for approximately $3.5 \%$ of the patients undergoing hemodialysis in Greece.

The inclusion criteria were the following: (a) age above 18 years, (b) undergoing HD three times/week for at least 6 months, (c) native language - Greek, (d) ability to read and sign the consent form, (e) time-space oriented, and (f) not currently undergoing transplant procedures. Patients with mental or cognitive disorders were excluded from the study.

Ethical consideration for the study was approved by the ethics committees of the six HD Units, and permission to conduct the study was also granted by the Greek data protection authority (Protocol number: $\Gamma$ N/E $\Xi / 4670-3 / 04-08-2016$ ). In addition, informed consent was obtained from all participants, who were ensured of the voluntary nature of their participation, the confidentiality involved, their anonymity, and their right to withdraw at any time during the study.

\section{Instruments}

Data were collected through an anonymous, self-completed questionnaire that consisted of two parts:

(1) The first part contained questions regarding demographics (age, gender), social attributes (marital status), and clinical information such as duration of dialysis and comorbidities. Additional information about religiosity was assessed using two questions in a four-point Likert scale (i) how religious are you (ii) how close to god are you . Current activity level was assessed using a fourpoint Likert scale, where 1 corresponded to no symptoms and fully active and 4 corresponded to the extent of symptom burden and inability to get out of bed."

(2) The Greek version of Missoula-Vitas Quality of Life Index-15 (MVQoL-15) scale was used to assess QoL. It is a 15item, self-reported, five-point Likert scale ("strongly agree" to "strongly disagree"), which was designed to gather information regarding patients' QoL, especially in advanced and chronic diseases. It was developed as a brief measure that can assess subjective QoL of patients that can be quickly interpreted by health professionals in order to assist them in identifying and addressing patients' concerns that affect QoL. It is divided into five domains, namely, Symptoms, Functionality, Interpersonal Relationships, Well-being, and Transcendence. It has a general question about the overall QoL. It was created by Byock and Merriman, and translated and validated in Greek by Theophilou et al. (12).

\section{Statistical analyses}

Empirical data of the study were processed with SPSS v. 22.0 for Windows (SPSS, Inc., Chicago, IL, USA). Descriptive and inferential statistical methods were applied. Mean, standard 
deviation, and relative (\%) frequencies were calculated. In addition, to investigate the effect of patient characteristics on QoL, student's $t$-test and one-way analysis of variance were used for the association between categorical and continuous variables, and Pearson's correlation coefficient for the correlation between continuous variables. All reported p-values were two-tailed, and the statistical significance level was set at 0.05 .

\section{Results}

Of the total participants, $62.1 \%$ were male, with a mean age $61.80 \pm 15.11 ; 67.3 \%$ were living in urban areas; $59.1 \%$ were married; $61 \%$ were retired; and the majority of the patients were Christian Orthodox (95.9\%). While 52.6\% reported additional health problems, the mean duration on dialysis was 5.69 years. Self-reported religiosity was $2.56 \pm 1.08$ and connection with God was $2.54 \pm 1.11$. Finally, the reported activity level was 2.46 \pm 1.09 . Regarding the reported QoL, the mean value of MVQoLI-15 (17.22, SD = 3.85) that is larger than the 15 value that corresponds to the median point of the response measurement scale (theoretical index range), indicating that the majority of patients had satisfactory total QoL values. The majority of the HD patients evaluated positively for the different dimensions of MVQoLI-15, with the exception of Well-being, which had mean and median values with a negative sign, that is, a negative evaluation. More specifically, Global QoL score was $3.41 \pm 0.93$; Symptoms domains, 4.39 \pm 9.95; Functionality, $6.59 \pm 10.56$; Interpersonal, $12.24 \pm 12.97$; Well-being, $-6.70 \pm 13.38$; Transcendence, $5.67 \pm 14.78$; and finally Total QoL, $17.22 \pm 3.85$. In Tables 1 and 2 are reporting the association between QoL domain and patient's characteristic (bivariate analysis).

Bivariate analysis revealed that the domain Global QoL was negatively associated with age $(\mathrm{p}<0.001)$, area of residence $(p=0.009)$, marital status $(p=0.001)$, number of children $(p=0.003)$, cohabitate $(p=0.019)$, educational level ( $p$ $<0.001)$, occupation $(\mathrm{p}<0.001)$, additional health problems $(\mathrm{p}<0.001)$, and current activity level $(\mathrm{p}<0.001)$. Symptoms domain was associated with marital status $(p=0.003)$, educational level $(p=0.032),(p<0.001)$, connection to god ( $p$ $=0.017)$, additional health problems $(\mathrm{p}<0.001)$, and current activity level $(\mathrm{p}<0.001)$. Functionality domain was only associated with area of residence $(p=0.001)$ and current activity level $(p=0.001)$. The domain Interpersonal relationships was found to be associated with marital status $(p<0.001)$, cohabitate $(p<0.001)$, religiosity $(p=0.009)$, connection to $\operatorname{god}(\mathrm{p}=0.002)$, additional health problems $(\mathrm{p}<0.001)$, and current activity level $(\mathrm{p}<0.001)$. Patients' well-being was associated only with current activity level ( $p<0.001)$. Transcendence domain was found to be associated with gender $(p=0.016)$, age $(p<0.001)$, area of residence $(p=0.049)$, marital status $(p=0.002)$, educational level $(p<0.001)$, additional health problems $(\mathrm{p}<0.001)$, and current activity level $(\mathrm{p}<0.001)$. Finally, total QoL domain was associated with age $(p=0.001)$, area of residence $(p=0.001)$, marital status $(p=0.002)$, cohabitate $(p=0.006)$, educational level $(p=0.003)$, connection to $\operatorname{god}(p=0.005)$, additional health problems $(\mathrm{p}<0.001)$, and current activity level $(\mathrm{p}<0.001)$.

\section{Discussion}

The aim of the present study was to assess QoL of ESRD patients undergoing hemodialysis. According to our results, patients reported satisfactory level of QoL in most of the domains that were assessed except for the Well-being domain. Most of the demographic, social and clinical factors appear to have an influence on QoL both as a total and individual domains. Among the socio-demographic factors, age, female gender, non-urban residence, lack of companionship and low educational attainment are associated with lower QoL. Concerning clinical features, comorbidity and high level of activity due to symptoms are associated with lower QoL, consistent with findings in the international literature (13-15).

In the present study, there was no relationship between sex and QoL, which contradicts existing research in international literature that claims women to report poorer QoL (16-18). Those findings could be attributed to the fact that women perform multiple roles and have lower levels of support. While the demands of day-to-day life and the complexity of the roles that women are called to embody today may be almost the same in all societies, the Greek society is characterized by an extended family type, which is a rich source of social support that can be a source of encouragement to face the challenges of life.

It is widely accepted that age is an important factor that is either positively or negatively related to patients' QoL. Patients who participated in the study and were older had a poorer QoL. Specifically, according to the results of the present study, age was negatively correlated with almost all dimensions of QoL. This may be because older people have more psychological and social problems. In addition, QoL is related not only to the course of the disease over time but also to the expected decline in aging (13-15).

In terms of marital status, it has been found that married patients have a better QoL than single people. It is well known that a family environment is a source of support. However, marital status has been the focus of several studies. On the one hand, there is a belief that family is a social support network (19), while, on the other hand, researchers argue that family obligations increase family anxiety, resulting in negative QoL (13).

Moreover, in this study, the level of education was found to be positively related to most of the dimensions of QoL. Specifically, secondary and tertiary graduates had higher levels of both overall QoL, as well as the transcendence and symptoms of MVQoLI-15. This finding strengthens existing studies that show QoL is positively correlated with patients' educational level $(14,20)$. In a study conducted in Turkey in 2009 , formal education graduates were found to have higher 
Table 1. Association of QoL and patient's characteristics $(n=367)$

\begin{tabular}{|c|c|c|c|c|}
\hline Characteristic & Global quality of life & Symptoms & Functionality & Interpersonal relationships \\
\hline \multicolumn{5}{|l|}{ Gender } \\
\hline Male & $3.48 \pm 0.89$ & $4.52 \pm 9.26$ & $6.10 \pm 11.16$ & $12.44 \pm 12.87$ \\
\hline Female & $3.31 \pm 0.99$ & $4.17 \pm 11.01$ & $7.40 \pm 9.48$ & $11.91 \pm 13.16$ \\
\hline $\mathrm{t}$ & 1.689 & 0.326 & 1.140 & 0.376 \\
\hline $\mathrm{p}$ & 0.092 & 0.745 & 0.255 & 0.707 \\
\hline \multicolumn{5}{|l|}{ Age } \\
\hline $\mathrm{r}$ & -0.202 & -0.097 & -0.004 & -0.039 \\
\hline $\mathrm{p}$ & $<0.001$ & 0.064 & 0.935 & 0.459 \\
\hline \multicolumn{5}{|l|}{ Place of residence } \\
\hline Urban area & $3.50 \pm 0.92$ & $4.85 \pm 10.47$ & $7.91 \pm 10.90$ & $12.93 \pm 13.61$ \\
\hline Non-urban area & $3.23 \pm 0.94$ & $3.45 \pm 8.75$ & $3.88 \pm 9.29$ & $10.82 \pm 11.45$ \\
\hline $\mathrm{t}$ & 2.615 & 1.263 & 3.487 & 1.558 \\
\hline $\mathrm{p}$ & 0.009 & 0.208 & 0.001 & 0.120 \\
\hline \multicolumn{5}{|l|}{ Marital status } \\
\hline Married & $3.55 \pm 0.91$ & $5.68 \pm 9.67$ & $6.01 \pm 11.69$ & $14.35 \pm 12.30$ \\
\hline Unmarried & $3.21 \pm 0.92$ & $2.53 \pm 10.08$ & $7.43 \pm 8.62$ & $9.18 \pm 13.33$ \\
\hline $\mathrm{t}$ & 3.489 & 3.016 & 1.342 & 3.772 \\
\hline $\mathrm{p}$ & 0.001 & 0.003 & 0.180 & $<0.001$ \\
\hline \multicolumn{5}{|l|}{ Children } \\
\hline Yes & $3.39 \pm 0.98$ & $4.26 \pm 10.79$ & $6.58 \pm 11.10$ & $12.97 \pm 12.93$ \\
\hline No & $3.47 \pm 0.80$ & $4.70 \pm 7.60$ & $6.63 \pm 9.18$ & $10.48 \pm 12.93$ \\
\hline $\mathrm{t}$ & 0.837 & 0.449 & 0.045 & 1.682 \\
\hline $\mathrm{p}$ & 0.404 & 0.654 & 0.964 & 0.093 \\
\hline \multicolumn{5}{|l|}{ Number of children } \\
\hline $\mathrm{r}$ & -0.156 & -0.046 & 0.032 & 0.074 \\
\hline $\mathrm{p}$ & 0.003 & 0.379 & 0.543 & 0.157 \\
\hline \multicolumn{5}{|l|}{ Living alone? } \\
\hline Yes & $3.21 \pm 1.04$ & $3.01 \pm 10.52$ & $7.71 \pm 8.24$ & $7.26 \pm 14.19$ \\
\hline No & $3.48 \pm 0.89$ & $4.81 \pm 9.74$ & $6.25 \pm 11.16$ & $13.77 \pm 12.19$ \\
\hline $\mathrm{t}$ & 2.347 & 1.471 & 1.315 & 3.842 \\
\hline $\mathrm{p}$ & 0.019 & 0.142 & 0.190 & $<0.001$ \\
\hline \multicolumn{5}{|l|}{ Educational status } \\
\hline Primary education (1) & $3.14 \pm 0.94$ & $2.92 \pm 11.65$ & $5.68 \pm 10.17$ & $10.86 \pm 13.50$ \\
\hline High school (2) & $3.59 \pm 0.86$ & $4.79 \pm 8.89$ & $6.91 \pm 10.19$ & $12.83 \pm 12.29$ \\
\hline University (3) & $3.59 \pm 0.94$ & $6.71 \pm 7.57$ & $7.86 \pm 12.22$ & $13.89 \pm 13.29$ \\
\hline
\end{tabular}

(Continued) 
Table 1. (Continued)

\begin{tabular}{|c|c|c|c|c|}
\hline Characteristic & Global quality of life & Symptoms & Functionality & Interpersonal relationships \\
\hline $\mathrm{F}$ & 10.929 & 3.463 & 1.063 & 1.494 \\
\hline $\mathrm{p}$ & $<0.001$ & 0.032 & 0.346 & 0.226 \\
\hline \multirow{2}{*}{$\begin{array}{l}\text { Post Hoc Test least } \\
\text { significant difference (LSD) }\end{array}$} & $(1)<(2) p<0.001$ & $(1)<(2) p=0.100$ & & \\
\hline & $(1)<(3) p=0.001$ & $(1)<(3) p=0.012$ & & \\
\hline \multicolumn{5}{|l|}{ Occupation } \\
\hline Working & $3.83 \pm 0.74$ & $7.36 \pm 6.46$ & $7.64 \pm 9.42$ & $13.90 \pm 11.54$ \\
\hline Not Working & $3.32 \pm 0.95$ & $3.69 \pm 10.49$ & $6.34 \pm 10.81$ & $11.85 \pm 13.27$ \\
\hline $\mathrm{t}$ & 4.913 & 3.731 & 0.926 & 1.299 \\
\hline $\mathrm{p}$ & $<0.001$ & $<0.001$ & 0.355 & 0.197 \\
\hline \multicolumn{5}{|l|}{ Religion } \\
\hline Christian Orthodox & $3.41 \pm 0.93$ & $4.37 \pm 9.99$ & $6.70 \pm 10.58$ & $12.22 \pm 12.97$ \\
\hline Not Christian Orthodox & $3.47 \pm 0.99$ & $4.87 \pm 9.19$ & $4.00 \pm 10.10$ & $12.73 \pm 13.24$ \\
\hline $\mathrm{t}$ & 0.223 & 0.189 & 0.971 & 0.150 \\
\hline $\mathrm{p}$ & 0.824 & 0.850 & 0.332 & 0.881 \\
\hline \multicolumn{5}{|l|}{ How religious are you? } \\
\hline $\mathrm{r}$ & 0.078 & 0.073 & 0.046 & 0.136 \\
\hline $\mathrm{p}$ & 0.138 & 0.160 & 0.381 & 0.009 \\
\hline \multicolumn{5}{|l|}{ How close to god are you? } \\
\hline $\mathrm{r}$ & 0.085 & 0.124 & 0.031 & 0.160 \\
\hline $\mathrm{p}$ & 0.105 & 0.017 & 0.556 & 0.002 \\
\hline \multicolumn{5}{|l|}{ Duration of dialysis } \\
\hline $\mathrm{r}$ & 0.005 & 0.042 & -0.077 & -0.097 \\
\hline $\mathrm{p}$ & 0.921 & 0.424 & 0.141 & 0.063 \\
\hline \multicolumn{5}{|l|}{ Additional health problems } \\
\hline Yes & $3.20 \pm 0.99$ & $2.45 \pm 10.58$ & $6.23 \pm 10.22$ & $10.02 \pm 13.10$ \\
\hline No & $3.66 \pm 0.80$ & $6.54 \pm 8.73$ & $6.99 \pm 10.94$ & $14.70 \pm 12.39$ \\
\hline $\mathrm{t}$ & 4.905 & 4.053 & 0.694 & 3.516 \\
\hline $\mathrm{p}$ & $<0.001$ & $<0.001$ & 0.488 & $<0.001$ \\
\hline \multicolumn{5}{|l|}{ Current activity level } \\
\hline $\mathrm{r}$ & 0.241 & 0.320 & 0.167 & 0.215 \\
\hline $\mathrm{p}$ & $<0.001$ & $<0.001$ & 0.001 & $<0.001$ \\
\hline
\end{tabular}

Data are presented as mean $\pm \mathrm{sd}$; Bold indicates significant $\mathrm{p}$ values. 
Table 2. Association of QoL and patient's characteristics $(n=367)$

\begin{tabular}{|c|c|c|c|}
\hline Characteristics & Well-being & Transcendence & Total QoL \\
\hline \multicolumn{4}{|l|}{ Gender } \\
\hline Male & $-6.43 \pm 13.07$ & $7.18 \pm 13.58$ & $17.38 \pm 3.70$ \\
\hline Female & $-7.14 \pm 13.91$ & $3.19 \pm 16.31$ & $16.95 \pm 4.09$ \\
\hline $\mathrm{t}$ & 0.492 & 2.415 & 1.031 \\
\hline $\mathrm{p}$ & 0.623 & 0.016 & 0.303 \\
\hline \multicolumn{4}{|l|}{ Age } \\
\hline $\mathrm{r}$ & -0.097 & -0.270 & -0.177 \\
\hline $\mathrm{p}$ & 0.063 & $<0.001$ & 0.001 \\
\hline \multicolumn{4}{|l|}{ Place of residence } \\
\hline Urban area & $-5.97 \pm 14.12$ & $6.64 \pm 15.88$ & $17.64 \pm 4.07$ \\
\hline Non-urban area & $-8.21 \pm 11.63$ & $3.68 \pm 12.03$ & $16.36 \pm 3.21$ \\
\hline $\mathrm{t}$ & 1.505 & 1.978 & 3.256 \\
\hline $\mathrm{p}$ & 0.133 & 0.049 & 0.001 \\
\hline \multicolumn{4}{|l|}{ Marital status } \\
\hline Married & $-6.33 \pm 13.75$ & $7.65 \pm 15.10$ & $17.74 \pm 3.87$ \\
\hline Unmarried & $-7.25 \pm 12.86$ & $2.81 \pm 13.87$ & $16.47 \pm 3.72$ \\
\hline $\mathrm{t}$ & 0.647 & 3.122 & 3.134 \\
\hline $\mathrm{p}$ & 0.518 & 0.002 & 0.002 \\
\hline \multicolumn{4}{|l|}{ Children } \\
\hline Yes & $-6.80 \pm 13.69$ & $5.32 \pm 15.66$ & $17.23 \pm 4.03$ \\
\hline No & $-6.46 \pm 12.66$ & $6.52 \pm 12.45$ & $17.19 \pm 3.41$ \\
\hline $\mathrm{t}$ & 0.222 & 0.779 & 0.102 \\
\hline $\mathrm{p}$ & 0.825 & 0.437 & 0.919 \\
\hline \multicolumn{4}{|l|}{ Number of children } \\
\hline $\mathrm{r}$ & -0.016 & -0.049 & -0.003 \\
\hline $\mathrm{p}$ & 0.763 & 0.349 & 0.961 \\
\hline \multicolumn{4}{|l|}{ Living alone? } \\
\hline Yes & $-8.86 \pm 13.23$ & $3.02 \pm 13.82$ & $16.21 \pm 3.77$ \\
\hline No & $-6.04 \pm 13.38$ & $6.48 \pm 14.99$ & $17.53 \pm 3.83$ \\
\hline $\mathrm{t}$ & 1.713 & 1.905 & 2.791 \\
\hline $\mathrm{p}$ & 0.087 & 0.058 & 0.006 \\
\hline \multicolumn{4}{|l|}{ Educational status } \\
\hline Primary education (1) & $-6.74 \pm 12.69$ & $1.44 \pm 14.97$ & $16.42 \pm 4.06$ \\
\hline High school (2) & $-7.15 \pm 14.34$ & $8.44 \pm 14.50$ & $17.58 \pm 3.62$ \\
\hline University (3) & $-5.48 \pm 12.50$ & $8.32 \pm 12.85$ & $18.13 \pm 3.66$ \\
\hline
\end{tabular}

(Continued) 
Table 2 . (Continued)

\begin{tabular}{|c|c|c|c|}
\hline Characteristics & Well-being & Transcendence & Total QoL \\
\hline $\mathrm{F}$ & 0.354 & 10.205 & 5.751 \\
\hline $\mathrm{p}$ & 0.702 & $<0.001$ & 0.003 \\
\hline \multirow[t]{2}{*}{ Post Hoc Test (LSD) } & & $(1)<(2)$ p $<0.001$ & $(1)<(2) p=0.008$ \\
\hline & & $(1)<(3) p=0.002$ & $(1)<(3) p=0.003$ \\
\hline \multicolumn{4}{|l|}{ Occupation } \\
\hline Working & $-4.09 \pm 13.23$ & $10.10 \pm 11.49$ & $18.49 \pm 3.33$ \\
\hline Not working & $-7.32 \pm 13.36$ & $4.63 \pm 15.29$ & $16.92 \pm 3.91$ \\
\hline $\mathrm{t}$ & 1.825 & 3.348 & 3.109 \\
\hline $\mathrm{p}$ & 0.069 & 0.001 & 0.002 \\
\hline \multicolumn{4}{|l|}{ Religion } \\
\hline Christian Orthodox & $-6.76 \pm 13.42$ & $5.47 \pm 14.89$ & $17.20 \pm 3.88$ \\
\hline Not Christian Orthodox & $-5.47 \pm 12.88$ & $10.33 \pm 11.34$ & $17.65 \pm 3.21$ \\
\hline $\mathrm{t}$ & 0.365 & 1.248 & 0.439 \\
\hline $\mathrm{p}$ & 0.715 & 0.213 & 0.661 \\
\hline \multicolumn{4}{|l|}{ How religious are you? } \\
\hline $\mathrm{r}$ & 0.014 & 0.001 & 0.082 \\
\hline $\mathrm{p}$ & 0.794 & 0.994 & 0.116 \\
\hline \multicolumn{4}{|l|}{ How close to god are you? } \\
\hline $\mathrm{r}$ & 0.060 & 0.084 & 0.148 \\
\hline $\mathrm{p}$ & 0.251 & 0.108 & 0.005 \\
\hline \multicolumn{4}{|l|}{ Duration of dialysis } \\
\hline $\mathrm{r}$ & 0.005 & 0.033 & -0.029 \\
\hline $\mathrm{p}$ & 0.929 & 0.532 & 0.582 \\
\hline \multicolumn{4}{|l|}{ Additional health problems } \\
\hline Yes & $-7.12 \pm 13.82$ & $3.13 \pm 15.53$ & $16.47 \pm 3.89$ \\
\hline No & $-6.24 \pm 12.89$ & $8.49 \pm 13.40$ & $18.05 \pm 3.64$ \\
\hline $\mathrm{t}$ & 0.627 & 3.548 & 3.997 \\
\hline $\mathrm{p}$ & 0.531 & $<0.001$ & $<0.001$ \\
\hline \multicolumn{4}{|l|}{ Current activity level } \\
\hline $\mathrm{r}$ & 0.182 & 0.406 & 0.420 \\
\hline $\mathrm{p}$ & $<0.001$ & $<0.001$ & $<0.001$ \\
\hline
\end{tabular}

Data are presented as mean $\pm \mathrm{sd}$; Bold indicates significant $\mathrm{p}$ values. 
scores, both in overall QoL and in the dimension of fitness and symptoms (20). In addition, according to the results of a recent study (21), educational level was positively correlated with psychosocial dimensions of QoL, which is confirmed by the present results, as the spiritual dimension of QoL was found to be influenced by the educational background of the participants. This may be due to the fact that those with a good educational background can more easily understand the nature of the disease and avoid aggravating factors and consequently have better QoL levels. However, this relationship between educational level and QoL is controversial as studies reject it (22).

According to the results of the present study, another factor that influences several dimensions is the place of residence. In particular, urban dwellers appear to have higher levels of QoL in terms of overall spirituality and functionality, compared to non-urban dwellers. This is another finding that is controversial in international literature, as there are studies that reinforce such claims (23) and others that refute them (24). One possible explanation for such controversial results is the disparities in healthcare systems across countries.

One factor that seems to have a positive effect on QoL in our results is the existence of work. A study by Parvan, Lakdizaji, Roshangar, \& Mostofi (25), which included 245 hemodialysis patients, found that those who reported being unemployed had lower levels of QoL than those who had a job. Similar to the study of Tel (20), the same findings were found, concluding that appropriate occupational status is an important factor that is effective in improving the treatment of patients undergoing dialysis (20).

Regarding clinical factors that appear to be related to QoL in CKD, the duration of dialysis has a negative effect on interpersonal relationships, which is in agreement with several studies on social life in CKD (26). It is widely accepted that CKD, and especially dialysis, has a negative impact on social lives, whether it be the marital, parental, or professional roles. In addition, the problematic self-image these patients usually have, coupled with dietary restrictions on food and drink, leads to reduced participation in family and social events.

Additional health problems also had a negative impact on overall QoL, and on the dimensions of symptoms and transcendence, as in the present study, the proportion reporting comorbidity with CKD was $52.6 \%$. This high rate of comorbidity is confirmed in other studies (27). High comorbidity has been found to affect QoL (28) adversely. In a prospective study conducted in China involving 179 end-stage renal patients, patients with comorbid conditions were found to have lower levels of QoL (29). Also, a cross-sectional study in Saudi Arabia, which included 205 patients undergoing dialysis, found that the presence of comorbid conditions had a negative impact on QoL. Peripheral vascular disease was associated with poorer levels of QoL (27).
Finally, the burden of symptomatic functionality appears to affect all dimensions of QoL adversely. Patients with CKD experience a number of symptoms that vary in intensity and affect the PH and potentially their functionality. According to researchers, symptom burden in combination with other clinical variables are key factors that lead to low QoL in CKD (30). The intensity and the number of symptoms have been found to negatively affect several dimensions of QoL, thus indicating the importance of effective symptom management in CKD (29).

\section{Conclusions}

The results of this study add to our knowledge on the QoL and associated factors of patients with CKD, as existing data from Greece are limited. The overall score for the MVQoL Index was above the median, which means that patients were reporting satisfactory QoL. In general, the role of patient characteristics in QoL is an area of interest, and early and proper intervention is important to enhance QoL, as well as to help these patients develop positive strategies for managing their illness, which will ultimately help them to slow the rate of disease progression and improve psychosocial outcomes.

\section{Conflicts of interest}

None.

\section{References}

1. Toulia G, Koutsopoulou V. Quality of life of Hemodialysis patients. Perioperative Nurs. 2015;4(1):10-18.

2. Zazzeroni L, Pasquinelli G, Nanni E, Cremonini V, Rubbi I. Comparison of quality of life in patients undergoing hemodialysis and peritoneal dialysis: A systematic review and meta-analysis. Kidney Blood Pres Res. 2017;42(4):717-27. https://doi. org/10.1159/000484115

3. Bello AK, Levin A, Lunney M. Global Kidney Health Atlas: A report by the International Society of Nephrology on the global burden of end-stage kidney disease and capacity for kidney replacement therapy and conservative care across world countries and regions. Brussels: International Society of Nephrology; 2019.

4. Karimi M, Brazie J. Health, health-related quality of life, and quality of life: What is the difference? PharmacoEconomics. 2016;34(7):645-9. https://doi.org/10.1007/s40273-016-0389-9

5. Gotay CC, Korn EL, McCabe MS, Moore TD, Cheson BD. Quality-of-life assessment in cancer treatment protocols: Research issues in protocol development. J Natl Cancer Inst. 1992;84:575-9. https://doi.org/10.1093/jnci/84.8.575

6. Schipper H, Clinch J. Assessment of treatment of cancer. In: Smith GT, editor. Measuring health: A practical approach. New York, NY: John Wiley \& Sons; 1988.

7. Theofilou P. Quality of life, mental health and health beliefs: Comparison between haemodialysis and peritoneal dialysis patient. Interscientific Health Care. 2010;2(4):171-6.

8. Heidenheim AP, Muirhead N, Moist L, Lindsay RM. Patient quality of life on quotidian hemodialysis. Am J Kidney Dis. 2003;42:36-41. https://doi.org/10.1016/S0272-6386(03)00536-5 
9. Theofilou T, Panagiotaki H. Factors influencing the quality of life of individuals with end-stage renal disease. Nosileftiki. 2010;49(2):174-81.

10. Ginieri-Coccossis M, Theofilou P, Synodinou C, Tomaras V, Soldatos C. Quality of life, mental health and health beliefs in haemodialysis and peritoneal dialysis patients: Investigating differences in early and later years of current treatment. BMC Nephrol. 2008;9:14. https://doi.org/10.1186/1471-2369-9-14

11. Leung KCD. Psychosocial aspects in renal patients. Perit Dial Int. 2003;23(S2):S90-4. https://doi.org/10.1177/089686080302302s19

12. Theofilou P, Aroni A, Ralli M, Gouzou M, Zyga S. Measuring health: Related quality of life in hemodialysis patients. Psychometric properties of the Missoula-VITAS Quality of Life Index (MVQOLI-15) in Greece. Health Psych Res. 2013;1(2):e17. https://doi.org/10.4081/hpr.2013.1004

13. Anees M, Malik MR, Abbasi T, Nasir Z, Hussain Y, Ibrahim M. Demographic factors affecting quality of life of hemodialysis patients-Lahore, Pakistan. Pakistan J Med Sci. 2014;30(5):1123-7. https://doi.org/10.12669/pjms.305.5239

14. Gerasimoula K, Lefkothea L, Maria L, Victoria A, Paraskevi T, Maria P. Quality of life in hemodialysis patients. Mater Sociomed. 2015;27(5):305-9. https://doi.org/10.5455/msm.2015.27.305-309

15. Zyga S. Chronic kidney disease and quality of life. Athens: Beta Publishing; 2017.

16. Santos P, Daher E, Silva G, Libório A, Kerr L. Quality of life assessment among haemodialysis patients in a single centre: A 2-year follow-up. Qual Life Res. 2009;18(5):541-6. https://doi. org/10.1007/s11136-009-9474-2

17. Mujais SK, Story K, Brouillette J, Takano T, Soroka S, Franek $\mathrm{C}$ et al. Health-related quality of life in CKD patients: Correlates and evolution over time. Clin J Am Soc Nephrol. 2009;4(8):1293-301. https://doi.org/10.2215/CJN.05541008

18. Hosny G, Kamil LA, Foda N. Assessment of quality of life for hemodialysis patients in Iraq. Int J Environ Sci Toxicol Res. 2017:5(1):10-22.

19. Abdelghany MA, Elgohary EE, Nienaa YA. Assessment of health-related quality of life in patients receiving regular hemodialysis. J Nephrol Ther. 2016;6:246. https://doi. org/10.4172/2161-0959.1000246

20. Tel H. Determining quality of life and sleep in hemodialysis patients. Dial Transplant. 2009;38(6):210-15. https://doi. org/10.1002/dat.20296
21. Zhou X, Xue F, Wang H, Qiao Y, Liu G, Huang L. et al. The quality of life and associated factors in patients on maintenance hemodialysis-A multicenter study in Shanxi province. Ren Fail. 2017;39(1):707-11. https://doi.org/10.1080/0886022x.2017.1398095

22. Bayoumi M, Al Wakeel J, Mishkiry A, Al Harbi A, Al Suwaida A, Al Ghonaim M. Predictors of quality of life in hemodialysis patients. Saudi J Kidney Dis Transplant. 2012;24(2):254. https:// doi.org/10.4103/1319-2442.109566

23. Yang S, Kuo P, Wang J, Lin M, Su S. Quality of life and its determinants of hemodialysis patients in Taiwan measured with WHOQOL-BREF (TW). Am J Kidney Dis. 2005;46(4):635-41. https://doi.org/10.1053/j.ajkd.2005.06.015

24. Saffari M, Pakpour A, Naderi M, Koenig H, Baldacchino D, Piper C. Spiritual coping, religiosity and quality of life: A study on Muslim patients undergoing haemodialysis. Nephrology. 2013;18(4):269-75. https://doi.org/10.1111/nep.12041

25. Parvan K, lakdizaji S, Roshangar F, Mostofi M. Quality of sleep and its relationship to quality of life in hemodialysis patients. J Caring Sci. 2013;2(4):295-304. https://doi.org/10.5681/ jes. 2013.035

26. Minos Ch, Rekleiti M, Saridi M, Filippopoulou T, Tzitzikos $\mathrm{G}$, Souliotis K, et al. Factors affecting quality of life in endstage renal disease patients on hemodialysis. Hellenic J Nurs Sci. 2012;5(2):64-71.

27. Mandoorah QM, Shaheen FA, Mandoora SM, Bawazi SA, Alshohaib SS. Impact of demographic and comorbid conditions on quality of life of hemodialysis patients: A cross-sectional study. Saudi J Kidney Dis Transplant. 2014;5(2):432. https://doi.org/10.4103/1319-2442.128613

28. Valderrábano F, Jofre R, López-Gómez J. Quality of life in endstage renal disease patients. Am J Kidney Dis. 2001;38(3):443-64. https://doi.org/10.1053/ajkd.2001.26824

29. Yong DS, Kwok AO, Wong DM, Suen MH, Chen WT, Tse DM. Symptom burden and quality of life in end-stage renal disease: A study of 179 patients on dialysis and palliative care. Palliat Med. 2009;23(2):111-19. https://doi. org/10.1177/0269216308101099

30. Alshammari B, Noble H, McAneney H, O'Halloran P. An exploration of symptom burden and its management, in Saudi Arabian patients receiving haemodialysis, and their caregivers: A mixed methods study protocol. BMC Nephrol. 2019;20(1):250. https://doi.org/10.1186/s12882-019-1424-9 